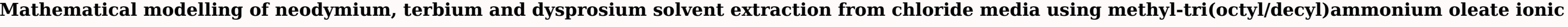 \\ liquid as extractant
}

E. Obón

A. Fortuny ${ }^{\mathrm{a}}$

M.T. Coll ${ }^{\mathrm{a}}$

A.M. Sastre ${ }^{b, *}$

ana.maria.sastre@upc.edu

${ }^{a}$ Chemical Engineering Department, Universitat Politècnica de Catalunya, EPSEVG, Av. Víctor Balaguer 1, 08800 Vilanova i la Geltrú, Spain

${ }^{\mathbf{b}}$ Chemical Engineering Department, Universitat Politècnica de Catalunya, ESTEIB, Av. Diagonal 647, 08028 Barcelona, Spain

${ }^{*}$ Corresponding author.

Abstract

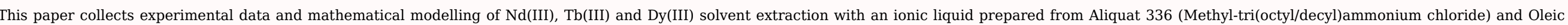

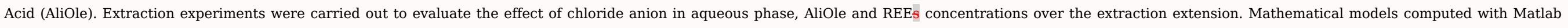

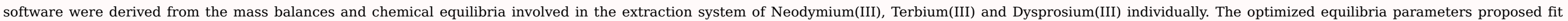
accurately the experimental data and allowed us to predict the extraction extension of each metal from an aqueous mixture.

Keywords: Neodymium; Terbium; Dysprosium; ițonic liquid; eExtraction model

\subsection{Introduction}

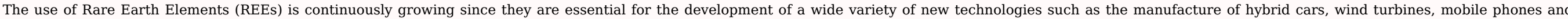

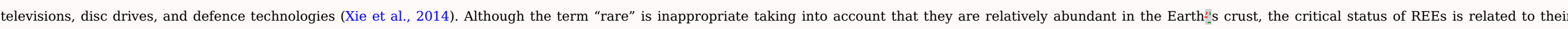

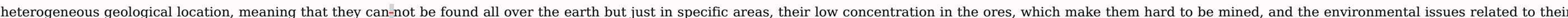
mining due to the high volume of concentrated acids needed to lixiviate them from the ores and the large amount of secondary waste generated after the process (European Comission, 2014).

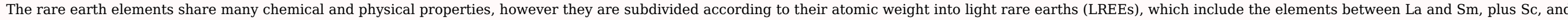
heavy rare earths (HREEs), which include the elements between Eu and Y.

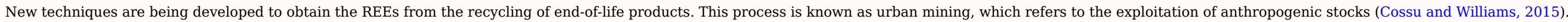

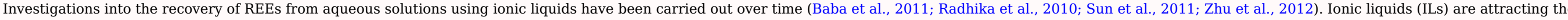

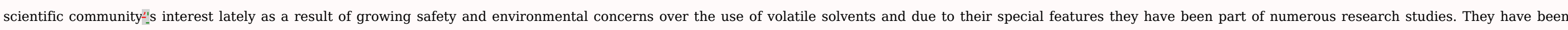

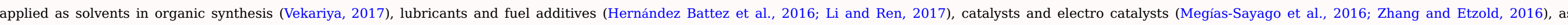

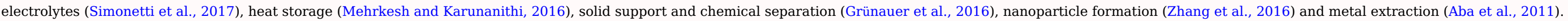

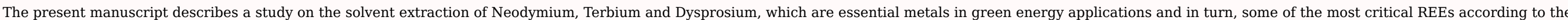




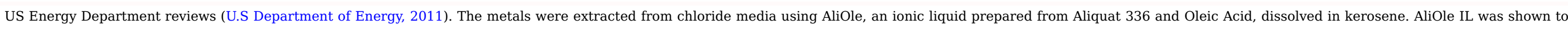
be promising for the extraction of REEs from aqueous solutions.

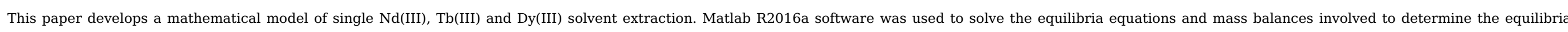

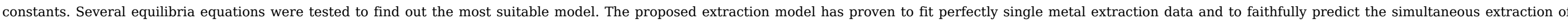
neodymium, terbium and dysprosium mixed in the aqueous phase. The validity of the approached equilibria constants was confirmed by comparing the plotted experimental and calculated data.

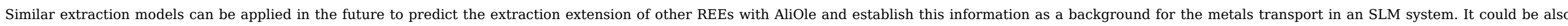
helpful to predict the potential separation factor between two rare earth elements at different experimental conditions.

\subsection{Experimental procedure}

\subsubsection{Reagents}

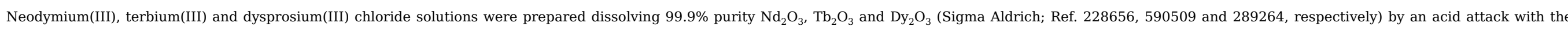
minimal amount of $\mathrm{HCl}$. $\mathrm{NaCl}$ was added when needed up to the required chloride concentration. The initial $\mathrm{pH}$ of the solutions was adjusted to 3.5 with $\mathrm{NaOH}$.

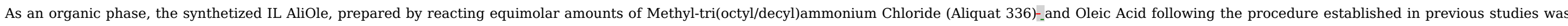
used (Coll et al., 2014; Fortuny et al., 2012). Kerosene was used as a diluent.

\subsubsection{Procedure}

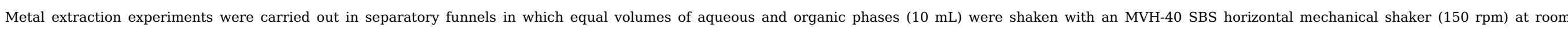

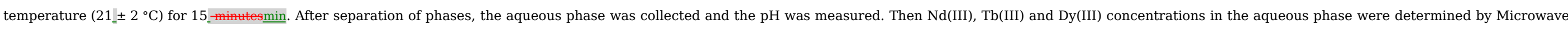
Plasma Atomic Emission Spectroscopy with an MP-AES 4100 spectrophotometer (Agilent Technologies).

The organic phase was kept for stripping experiments. Hydrochloric acid $1 \mathrm{~mol} \cdot \mathrm{L}^{=1}$ was used to strip the REEs from the organic phase by three contacts.

Extraction experiments were performed using AliOle dissolved in kerosene from 0.005 to $0.1 \mathrm{~mol} \cdot \mathrm{L}^{=1}$ as organic phase and aqueous solutions containing $1000 \mathrm{mg} / \mathrm{L}$ of $\mathrm{Nd}(\mathrm{III})$, Tb(III) and Dy(III)

The effect of the AliOle concentration, the chloride concentration on the aqueous phase and the competitive extraction of the metals were studied.

\subsection{Mathematical modelling}

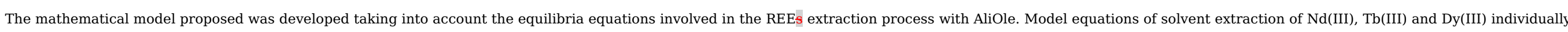

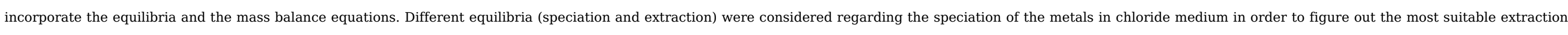
model.

\subsubsection{Equilibria equations}

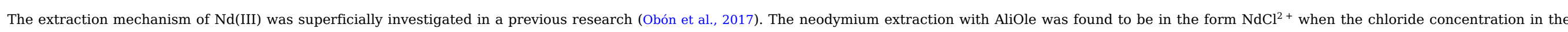

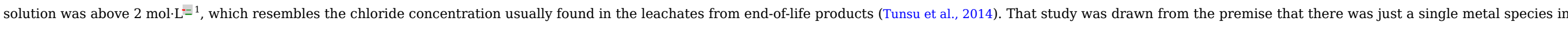

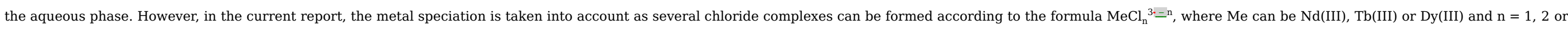
3 depending on the number of chlorides bonded with the metal ion. Therefore, the REEs distribution in the aqueous phase can be described as follows:

$$
\mathrm{Me}^{3+}+\mathrm{Cl}^{-} \stackrel{\mathrm{K}_{1}}{\leftrightarrow} \mathrm{MeCl}^{2+}
$$

$\mathrm{MeCl}^{2+}+\mathrm{Cl}^{-} \stackrel{\mathrm{K}_{2}}{\leftrightarrow} \mathrm{MeCl}_{2}^{+}$ 
$\mathrm{MeCl}_{2}+\mathrm{Cl}^{+} \stackrel{\mathrm{K}_{3}}{\leftrightarrow} \mathrm{MeCl}_{3}$

Wwhere Me can be $\mathrm{Nd}(\mathrm{III})$, $\mathrm{Tb}(\mathrm{III})$ or $\mathrm{Dy}(\mathrm{III})$ and $\mathrm{K}_{1}, \mathrm{~K}_{2}$ and $\mathrm{K}_{3}$ represent the stepwise stability constants.

The equilibria expressions of the complex formation constants expressed as a function of the activities of the species $\left(\mathrm{a}_{\mathrm{i}}\right)$ are listed below:

$\mathrm{K}_{1}=\frac{\mathrm{a}_{\mathrm{MeCl}^{2+}}}{\mathrm{a}_{\mathrm{Me}^{3+} \cdot \mathrm{a}_{\mathrm{Cl}^{-}}}}=\frac{\left[\mathrm{MeCl}^{2+}\right]}{\left[\mathrm{Me}^{3+}\right] \cdot\left[\mathrm{Cl}^{-}\right]} \cdot \frac{\gamma_{\mathrm{MeCl}^{2+}}}{\gamma_{\mathrm{Me}^{3+} \cdot \gamma_{\mathrm{Cl}^{-}}}}$

$\mathrm{K}_{2}=\frac{\mathrm{a}_{\mathrm{MeCl}_{2}+}}{\mathrm{a}_{\mathrm{MeCl}^{2+}} \mathrm{a}_{\mathrm{Cl}^{-}}}=\frac{\left[\mathrm{MeCl}_{2}^{+}\right]}{\left[\mathrm{MeCl}^{2+}\right] \cdot\left[\mathrm{Cl}^{-}\right]} \cdot \frac{\gamma_{\mathrm{MeCl}_{2}+}}{\gamma_{\mathrm{MeCl}^{2+} \cdot \gamma_{\mathrm{Cl}^{-}}}}$

$\mathrm{K}_{3}=\frac{\mathrm{a}_{\mathrm{MeCl}_{3}}}{\mathrm{a}_{\mathrm{MeCl}_{2}+} \mathrm{a}_{\mathrm{Cl}^{-}}}=\frac{\left[\mathrm{MeCl}_{3}\right]}{\left[\mathrm{MeCl}_{2}{ }^{+}\right] \cdot\left[\mathrm{Cl}^{-}\right]} \cdot \frac{\gamma_{\mathrm{MeCl}_{3}}}{\gamma_{\mathrm{MeCl}_{2}+} \cdot \gamma_{\mathrm{Cl}^{-}}}$

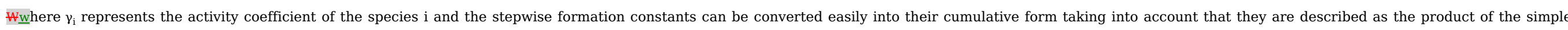
ones: $\beta_{1}=K_{1} ; \beta_{2}=K_{1} \cdots K_{2} ; \beta_{3}=K_{1} \cdots K_{2} \cdots K_{3}$.

The ionic strength of the metals was calculated from the equation:

$\mathrm{I}_{\mathrm{m}}=0.5 \sum \mathrm{z}_{\mathrm{i}}^{2} \mathrm{c}_{\mathrm{i}}$

$W_{\underline{w}}$ here $c_{i}$ is the concentration and $z_{i}$ is the charge of the species $i$.

The Davies equation was used to calculate the activity coefficients of the species in the aqueous phase when $I_{m} \neq 0$ :

$\log _{10} \gamma_{\mathrm{i}}=-0.5102 \cdot \mathrm{z}_{\mathrm{i}}\left(\frac{\sqrt{\mathrm{I}_{\mathrm{m}}}}{\left(1+\sqrt{\mathrm{I}_{\mathrm{m}}}\right)}-0.3 \cdot \mathrm{I}_{\mathrm{m}}\right)$

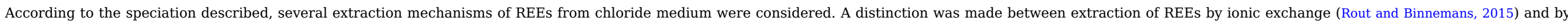

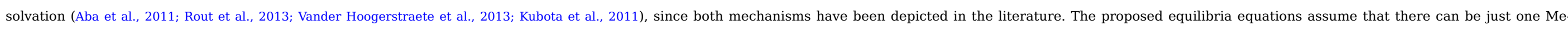

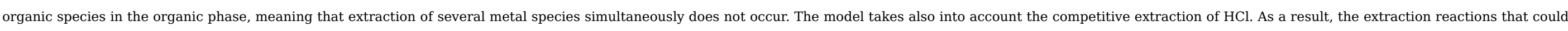
take place in this separation system of a single metal with the AliOle IL are listed below:

$3 \mathrm{R}_{4} \mathrm{~N}^{+} \mathrm{A}^{-}+\mathrm{Me}^{3+}+3 \mathrm{Cl}^{-} \stackrel{\mathrm{K}_{\mathrm{a}}}{\leftrightarrow} 3 \mathrm{R}_{4} \mathrm{~N}^{+} \mathrm{Cl}^{-}+\mathrm{MeA}_{3} 3 \overline{\mathrm{R}_{4} \mathrm{~N}^{+} \mathrm{A}^{-}}+\mathrm{Me}^{3+}+3 \mathrm{Cl}^{-} \stackrel{\mathrm{K}_{a}}{\leftrightarrow} 3 \overline{\mathrm{R}_{4} \mathrm{~N}^{+} \mathrm{Cl}^{-}}+\overline{\mathrm{MeA}_{3}}$

\begin{tabular}{|l|l|l}
\hline $\mathrm{R}_{4} \mathrm{~N}^{+} \mathrm{A}^{-}+\mathrm{Me}^{3+}+3 \mathrm{Cl}^{-} \stackrel{\mathrm{K}_{\mathrm{b}}}{\leftrightarrow}\left(\mathrm{R}_{4} \mathrm{~N}^{+} \mathrm{Cl}^{-}\right)_{3} \cdot \mathrm{MeA}_{3}$ & $3 \overline{\mathrm{R}_{4} \mathrm{~N}^{+} \mathrm{A}^{-}}+\mathrm{Me}^{3+}+3 \mathrm{Cl}^{-} \stackrel{\mathrm{K}_{\mathrm{b}}}{\leftrightarrow} \overline{\left(\mathrm{R}_{4} \mathrm{~N}^{+} \mathrm{Cl}^{-}\right)_{3} \cdot \mathrm{MeA}_{3}}$ \\
\hline
\end{tabular}

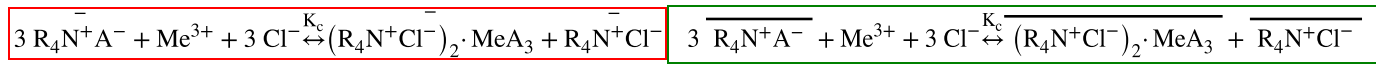

$3 \mathrm{R}_{4} \mathrm{~N}^{+} \mathrm{A}^{-}+\mathrm{Me}^{3+}+3 \mathrm{Cl}^{-} \stackrel{\mathrm{K}_{\mathrm{d}}}{\leftrightarrow} \mathrm{R}_{4} \mathrm{~N}^{+} \mathrm{Cl}^{-} \cdot \mathrm{MeA}_{3}+2 \mathrm{R}_{4} \mathrm{~N}^{+} \mathrm{Cl}^{-} 3 \overline{\mathrm{R}_{4} \mathrm{~N}^{+} \mathrm{A}^{-}}+\mathrm{Me}^{3+}+3 \mathrm{Cl}^{-} \stackrel{\mathrm{K}_{\mathrm{d}}}{\leftrightarrow} \overline{\mathrm{R}_{4} \mathrm{~N}^{+} \mathrm{Cl}^{-} \cdot \mathrm{MeA}_{3}}+2 \overline{\mathrm{R}_{4} \mathrm{~N}^{+} \mathrm{Cl}^{-}}$

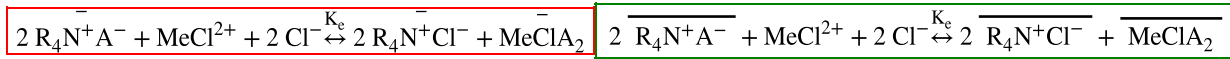

\begin{tabular}{|l|l|l}
$2 \mathrm{R}_{4} \mathrm{~N}^{+} \mathrm{A}^{-}+\mathrm{MeCl}^{2+}+2 \mathrm{Cl}^{-} \stackrel{\mathrm{K}_{\mathrm{f}}}{\leftrightarrow}\left(\mathrm{R}_{4} \mathrm{~N}^{+} \mathrm{Cl}^{-}\right)_{2} \cdot \mathrm{MeClA}_{2}$ & $2 \overline{\mathrm{R}_{4} \mathrm{~N}^{+} \mathrm{A}^{-}}+\mathrm{MeCl}^{2+}+2 \mathrm{Cl}^{-} \stackrel{\mathrm{K}_{\mathrm{f}}}{\leftrightarrow} \overline{\left(\mathrm{R}_{4} \mathrm{~N}^{+} \mathrm{Cl}^{-}\right)_{2} \cdot \mathrm{MeClA}_{2}}$ \\
\hline
\end{tabular} 
With the corresponding equilibria equations written as follows:

\begin{tabular}{|c|c|c|c|c|c|c|c|c|}
\hline${ }_{\mathrm{R}_{4} \mathrm{~N}^{+} \mathrm{Cl}^{-}}^{3^{3}} \cdot{ }_{\mathrm{MeA}_{3}}^{a_{-}^{-}}$ & {$\left[\mathrm{R}_{4} \mathrm{~N}^{+} \mathrm{Cl}^{-}\right.$} & $\cdot\left[\mathrm{MeA}_{3}^{-}\right]$ & $\gamma_{\mathrm{R}_{4} \mathrm{~N}^{+} \mathrm{Cl}^{-}}^{3} \cdot \gamma_{\mathrm{MeA}_{3}}^{-}$ & $\frac{\mathrm{a}}{\mathrm{R}_{4} \mathrm{~N}^{+} \mathrm{Cl}^{-}}$ & $\sqrt{\mathrm{MeA}_{3}}$ & $\mathrm{R}_{4} \mathrm{~N}^{+} \mathrm{Cl}^{-}$ & $\overline{\mathrm{MeA}_{3}}$ & $\gamma^{3} \overline{\mathrm{R}_{4} \mathrm{~N}^{+} \mathrm{Cl}^{-}} \cdot \gamma \overline{\mathrm{MeA}_{3}}$ \\
\hline$=\overline{\mathrm{a}_{\mathrm{R}_{4} \mathrm{~N}^{+} \mathrm{A}^{-}}} \cdot \mathrm{a}_{\mathrm{Me}^{3+}} \cdot \mathrm{a}_{\mathrm{Cl}}^{3}$ & $\left.\mathrm{R}_{4} \mathrm{~N}^{+} \mathrm{A}^{-}\right]^{3}$. & $\left.\mathrm{e}^{3+}\right] \cdot\left[\mathrm{Cl}^{-}\right]^{3}$ & $\gamma_{\mathrm{R}_{4} \mathrm{~N}^{+} \mathrm{A}^{-}} \cdot \gamma_{\mathrm{Me}^{3+} \cdot \gamma_{\mathrm{Cl}^{-}}^{3}}$ & $\mathrm{~K}_{\mathrm{a}}=\frac{}{\mathrm{a}^{3} \frac{}{\mathrm{R}_{4} \mathrm{~N}^{+} \mathrm{A}^{-}}}$ & $\mathrm{a}_{\mathrm{Me}^{3+} \cdot \mathrm{a}_{\mathrm{Cl}}{ }^{3}}$ & $\overline{\mathrm{R}_{4} \mathrm{~N}^{+} \mathrm{A}^{-}}$ & $\left.\mathrm{Ce}^{3+}\right] \cdot\left[\mathrm{Cl}^{-}\right]^{3}$ & 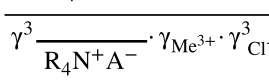 \\
\hline
\end{tabular}

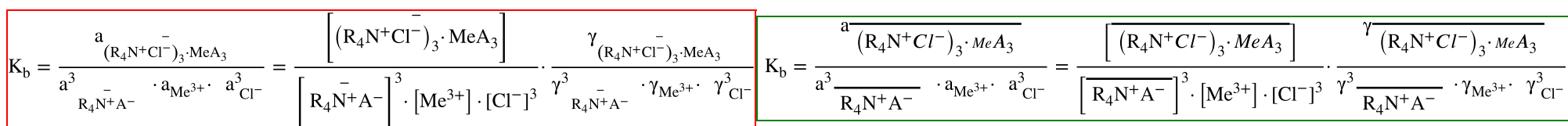

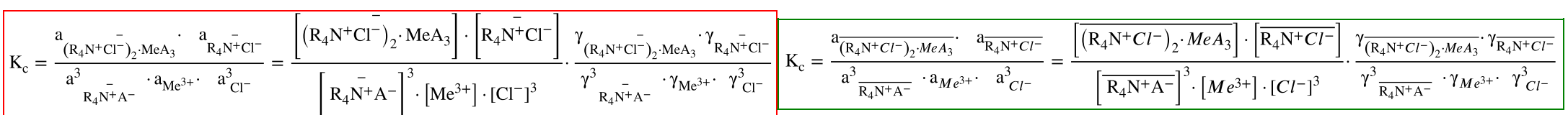

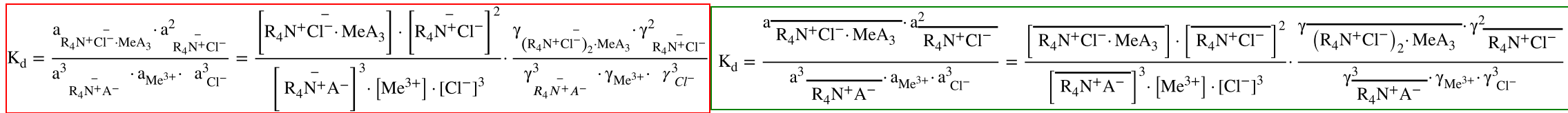

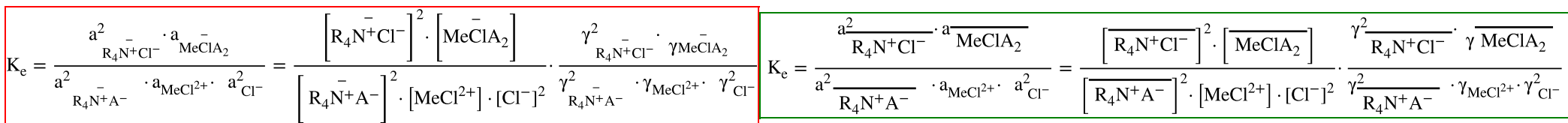

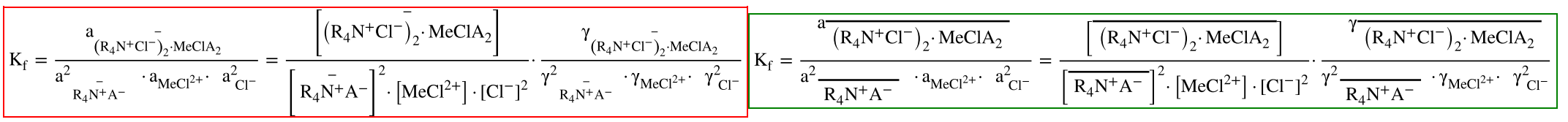

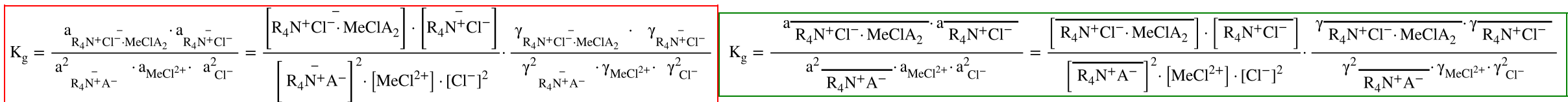

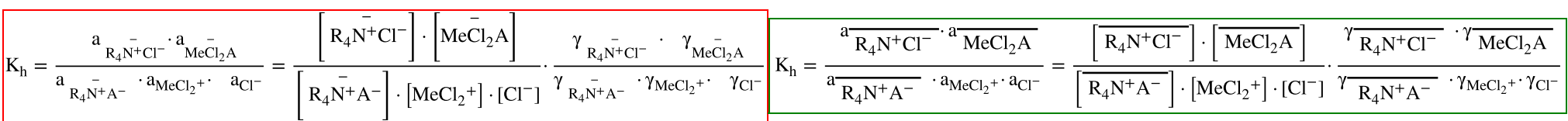




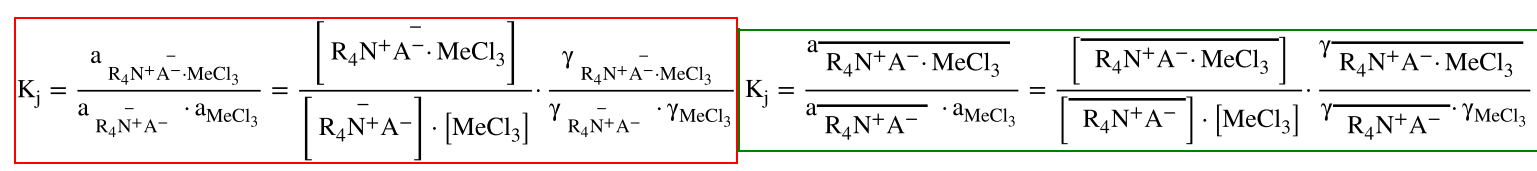

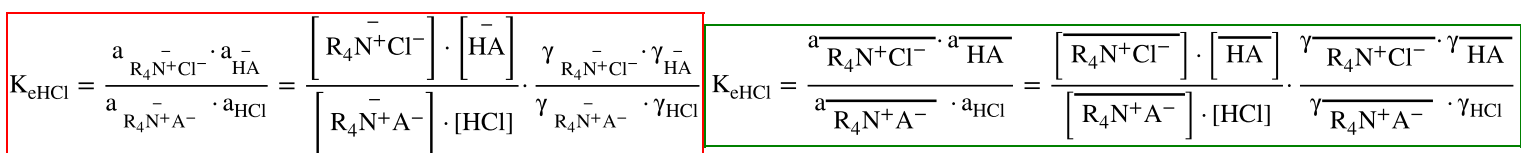

The bar above the species denotes that they are in the organic phase.

In the equilibria equations, the activity coefficients $(\gamma)$ of the organic and the aqueous neutral species are considered equal to 1.

\subsubsection{Mass balances}

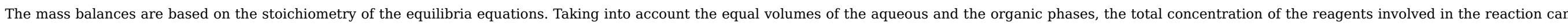
be calculated as the sum of the species present in the system. Generalized mass balances for the equilibria of single extraction reactions (R.4-R.13) can be written as follows:

REEs mass balance

$\left[\mathrm{Me}_{\mathrm{t}}=\left[\mathrm{Me}^{3+}\right]+\left[\mathrm{MeCl}^{2+}\right]+\left[\mathrm{MeCl}_{2}^{+}\right]+\left[\mathrm{MeCl}_{3}\right]+\left[\mathrm{Me}_{\text {org }}\right.\right.$

Chloride mass balance

$\left[\mathrm{Cl}^{-}\right]_{\mathrm{t}}=\left[\mathrm{MeCl}^{2+}\right]+2 \cdot\left[\mathrm{MeCl}_{2}{ }^{+}\right]+3 \cdot\left[\mathrm{MeCl}_{3}\right]+3 \cdot[\mathrm{Me}]_{\text {org }}+\Delta\left[\mathrm{H}^{+}\right]$

AliOle mass balance

$[\overline{\mathrm{E}}]_{\mathrm{t}}=[\overline{\mathrm{E}}]_{\text {free }}+\mathrm{m} \cdot[\mathrm{Me}]_{\text {org }}+\Delta\left[\mathrm{H}^{+}\right]$

Wwhere the subscript t means the total concentration of the specie, which in turn is the initial concentration, org represents the concentration of the species in the organic phase,

$[\overline{\mathrm{E}}]_{\text {free }}[\overline{\mathrm{E}}]_{\text {free }}$ is the extractant concentration available after $\mathrm{HCl}$ and metal extraction, $\Delta\left[\mathrm{H}^{+}\right]$is equal to the concentration of $\mathrm{HCl}$ in the organic phase and $\mathrm{m}$ can be 1,2 or 3 depending on the stoichiometry of the equilibria reaction considered.

\subsubsection{Competitive extraction prediction}

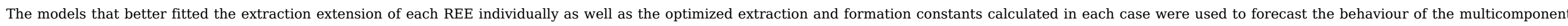
extraction process.

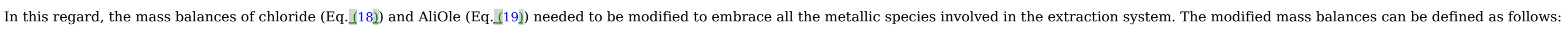

Nd(III) mass balance

$[\mathrm{Nd}]_{\mathrm{t}}=\left[\mathrm{Nd}^{3+}\right]+\left[\mathrm{NdCl}^{2+}\right]+\left[\mathrm{NdCl}_{2}^{+}\right]+\left[\mathrm{NdCl}_{3}\right]+[\mathrm{Nd}]_{\text {org }}$

$\mathrm{Tb}(\mathrm{III})$ mass balance

$[\mathrm{Tb}]_{\mathrm{t}}=\left[\mathrm{Tb}^{3+}\right]+\left[\mathrm{TbCl}^{2+}\right]+\left[\mathrm{TbCl}_{2}^{+}\right]+\left[\mathrm{TbCl}_{3}\right]+[\mathrm{Tb}]_{\mathrm{org}}$

Dy(III) mass balance

$[\mathrm{Dy}]_{\mathrm{t}}=\left[\mathrm{Dy}^{3+}\right]+\left[\mathrm{DyCl}^{2+}\right]+\left[\mathrm{DyCl}_{2}^{+}\right]+\left[\mathrm{DyCl}_{3}\right]+[\mathrm{Dy}]_{\text {org }}$

Chloride mass balance 
AliOle mass balance

$[\overline{\mathrm{E}}]_{\mathrm{t}}=[\overline{\mathrm{E}}]_{\text {free }}+\mathrm{m} \cdot[\mathrm{Ndd}]_{\text {org }}+\mathrm{n} \cdot[\mathrm{Tb}]_{\text {org }}+\mathrm{p} \cdot[\mathrm{Dy}]_{\text {org }}+\Delta\left[\mathrm{H}^{+}\right]$

Wwhere $\mathrm{n}$ and $\mathrm{p}$ can be 1,2 or 3 depending on the stoichiometry of the equilibria reaction considered.

For the competitive extraction prediction Eq.(23) is used instead of Eq._(18) and Eq._(24) is used instead of Eq._(19).

\section{Model resolution}

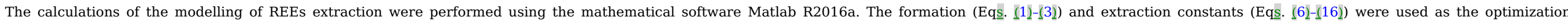
parameters in order to find the values that make experimental and calculated data match with the minimum error. The resolution method applied is shown in Figure. 1.

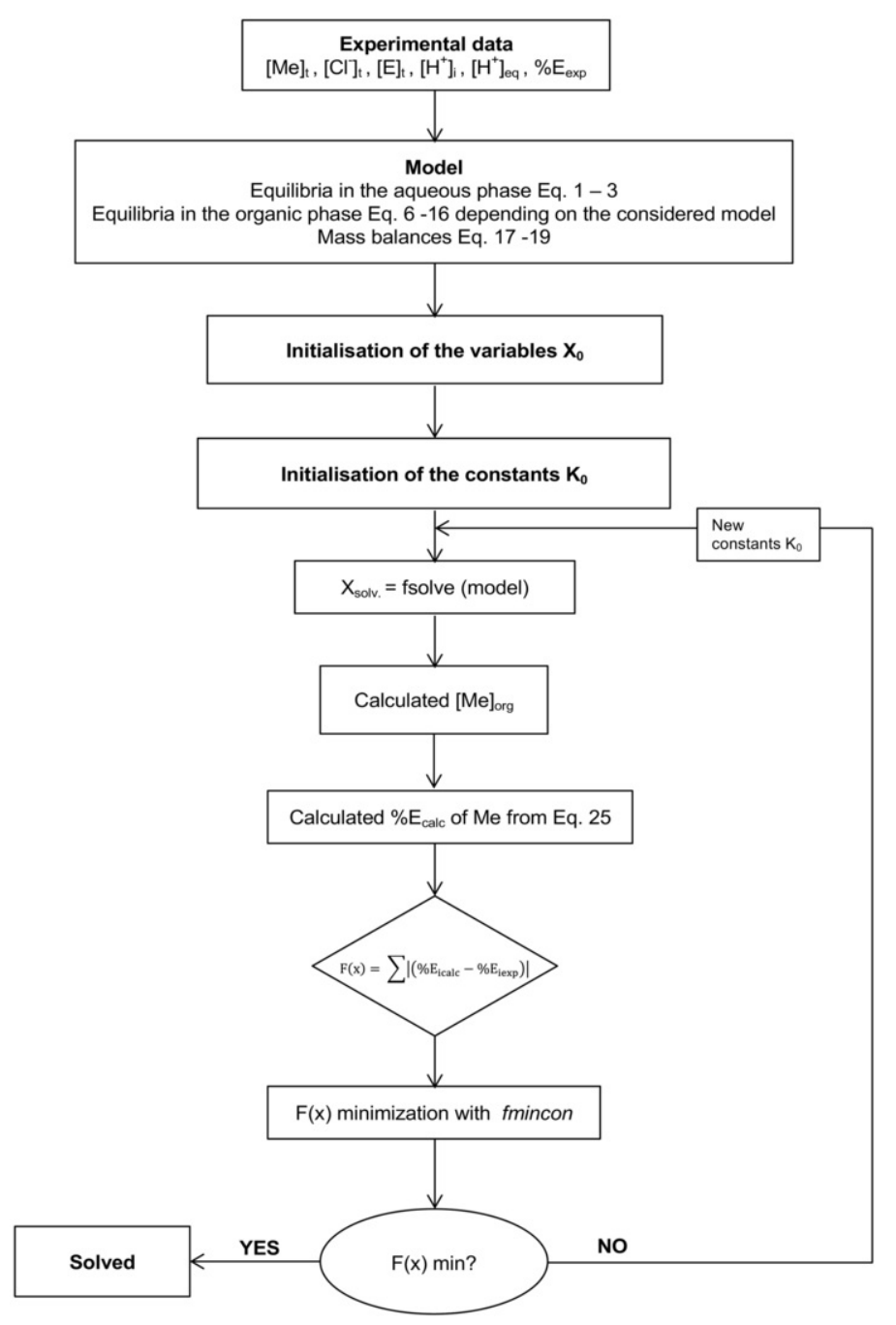




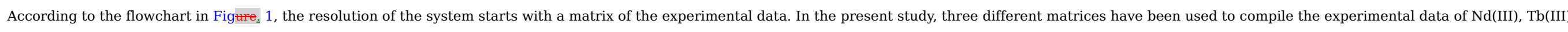

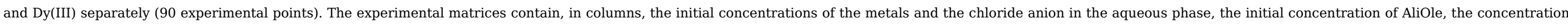

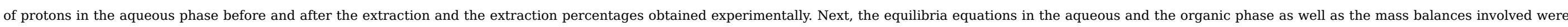

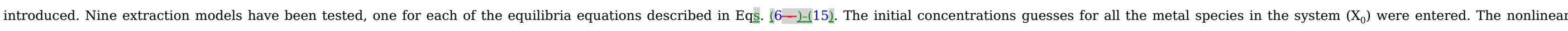

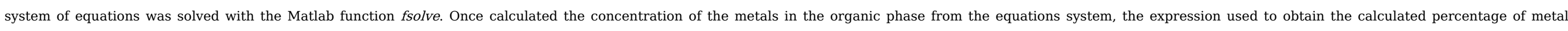
extraction is given as:

$$
\% \mathrm{E}_{\text {calc }}=\frac{[\mathrm{Me}]_{\text {org }}}{[\mathrm{Me}]_{\mathrm{t}}} \cdot 100
$$

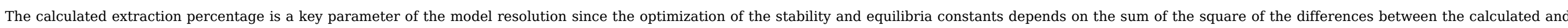
the experimental percentages of extraction.

Finally, the function $\mathrm{F}(\mathrm{x})$, defined as:

$$
\mathrm{F}(\mathrm{x})=\sum\left|\left(\% \mathrm{E}_{\text {icalc }}-\% \mathrm{E}_{\text {iexp }}\right)\right|
$$

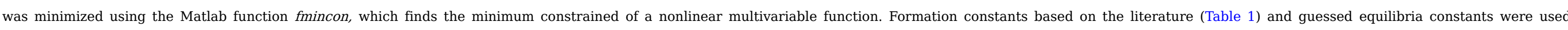
to initialize the program $\left(\mathrm{K}_{0}\right)$.

\begin{tabular}{|c|c|c|c|c|c|c|c|c|c|}
\hline & \multicolumn{3}{|c|}{ Nd(III) } & \multicolumn{3}{|c|}{$\operatorname{Tb}(\mathrm{III})$} & \multicolumn{3}{|c|}{ Dy(III) } \\
\hline & $\mathrm{K}_{1}$ & $\mathrm{~K}_{2}$ & $\mathrm{~K}_{3}$ & $\mathrm{~K}_{1}$ & $\mathrm{~K}_{2}$ & $\mathrm{~K}_{3}$ & $\mathrm{~K}_{1}$ & $\mathrm{~K}_{2}$ & $\mathrm{~K}_{3}$ \\
\hline $\mathrm{a}$ & 2.09 & 0.50 & $-=$ & 1.86 & 0.45 & $=$ & 1.86 & 0.45 & $-=$ \\
\hline $\mathrm{b}$ & 1.00 & $-=$ & $-=$ & $-=$ & $-=$ & $-=$ & $-=$ & $-=$ & $=$ \\
\hline c & $8.32 .10^{-=}=3$ & $=$ & $=$ & $=$ & $=$ & $=$ & $=$ & $=$ & $=$ \\
\hline $\mathrm{d}$ & 1.8 & 1.1 & $=$ & I= & $=$ & $=$ & $=$ & $=$ & $=$ \\
\hline
\end{tabular}

Table 1 Stability constants in the literature:

a. Medusa Software, KHT Royal Institute of Technology (Puigdomenech, 2013).
b. (Hogfeldt, 1982).
c. (Lee et al., 2005).
d. (Sastri, 2003).

The optimization led to the determination of the stability and equilibria constants, whose values allow the experimental extraction extension of the metals to be reproduced with the minimum error.

\section{5. $\underline{5}$ Results and discussion}

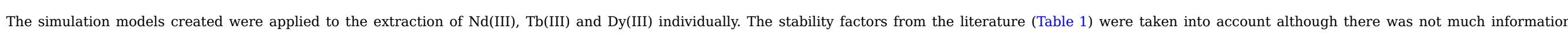

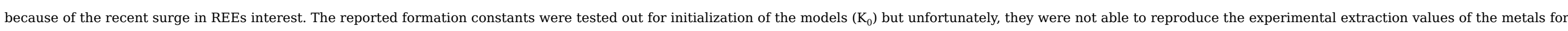
any of the proposed models. In this regard, it became necessary to find new constants beyond the literature, from scratch, in order to adjust the models to the experimental data of REEs solvent extraction. 


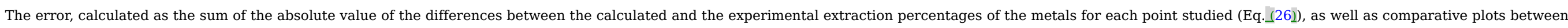

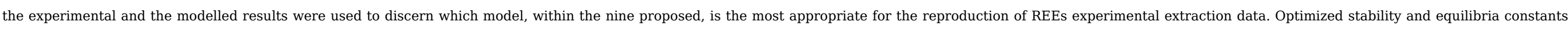
were determined in each case. Table 2 shows the optimized stability and extraction constants and the error value calculated for each model tested.

Table 2 Optimization parameters.

alt-text: Table 2

\begin{tabular}{|c|c|c|c|c|c|c|c|c|c|c|c|c|c|}
\hline & \multicolumn{4}{|c|}{$\mathrm{Nd}(\mathrm{III})$} & \multicolumn{4}{|c|}{$\mathrm{Tb}(\mathrm{III})$} & \multicolumn{4}{|c|}{ Dy(III) } & \multirow[t]{2}{*}{$\sigma$ total } \\
\hline & $\mathrm{K}_{\mathrm{eq}}$ & $\mathrm{K}_{1}$ & $\mathrm{~K}_{2}$ & $\mathrm{~K}_{3}$ & $\mathrm{~K}_{\mathrm{eq}}$ & $\mathrm{K}_{1}$ & $\mathrm{~K}_{2}$ & $\mathrm{~K}_{3}$ & $\mathrm{~K}_{\mathrm{eq}}$ & $\mathrm{K}_{1}$ & $\mathrm{~K}_{2}$ & $\mathrm{~K}_{3}$ & \\
\hline Eq. (6-)(a) & 7.88 & 0.09 & 0.94 & 0.13 & 7.62 & $1.20 .10^{-}=3$ & 0.24 & 0.69 & 6.79 & 0.16 & 0.33 & 0.06 & 8140.10 \\
\hline Eq. (7)-(b) & 50,223 & 0.75 & 1.17 & 0.72 & 63,541 & $-5.10^{-}=3$ & 0.43 & 0.27 & $50 \_965$ & 1.94 & 1.92 & 1.90 & 5492 \\
\hline Eq. (82-(c) & 7837.70 & 0.44 & $1.00 .10^{-}=5$ & 0.07 & 13,580 & $3.00 .10^{-}=3$ & 0.84 & 0.105 & 7737.9 & 4.363 & 0.732 & 0.13 & 4746.70 \\
\hline Eq. (9)-(d) & 82.39 & 0.57 & 0.44 & 0.49 & 1850.90 & 0.67 & 1.84 & 1.05 & 2321.60 & 1.67 & 0.36 & 61.15 & 7345.80 \\
\hline Eq. $(10)-(e)$ & 3.54 & 3.25 & 124.12 & 0.32 & 1.34 & 0.05 & 0.03 & 0.81 & 3.21 & 20.48 & 2.44 & 2.68 & 2347 \\
\hline Eq. $(11)(f)$ & 4223.02 & 37.14 & 0.15 & $9.50 .10^{-}=4$ & 5573.10 & 252.97 & 0.03 & 0.30 & 7259.50 & 98.39 & 0.02 & 1.00 & 599.25 \\
\hline Eq. $(12)(\mathrm{g})$ & 5559.30 & 87.22 & 0.10 & $7.65 .10^{-}=4$ & 6515.50 & 84.04 & 0.08 & $4.72 .10^{-}=6$ & 5021.90 & 100.59 & $3.30 .10^{-}=3$ & 1.76 & 652.95 \\
\hline Eq. $(13)-(h)$ & 0.08 & 71.25 & 1.51 & 0.02 & 41.99 & 306.84 & 6.71 & 0.87 & 3.92 & 75.04 & 0.01 & $1.10 .10^{-}=4$ & 3239 \\
\hline Eq. (14)-(i) & 53.67 & 39.66 & 149.98 & 0.12 & 56.58 & 253.39 & 25.73 & 0.16 & 84.11 & 99.17 & 18.89 & 0.42 & 1428 \\
\hline Eq. $(15)-(j)$ & 435.17 & 34.27 & 155.78 & 0.12 & 356.59 & 249.44 & 18.15 & 0.16 & 198.31 & 110.08 & 0.18 & 42.50 & 1427.60 \\
\hline
\end{tabular}

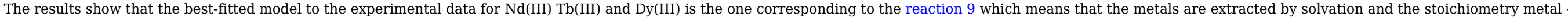
IL is $1: 2$ :

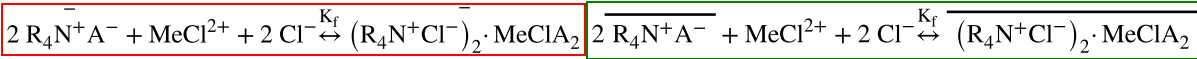

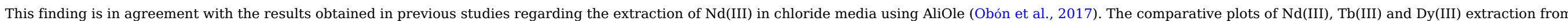

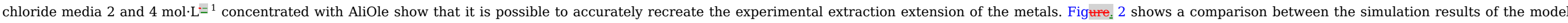
obtained by using the optimized parameters calculated with Matlab software and the experimental extraction percentages. 

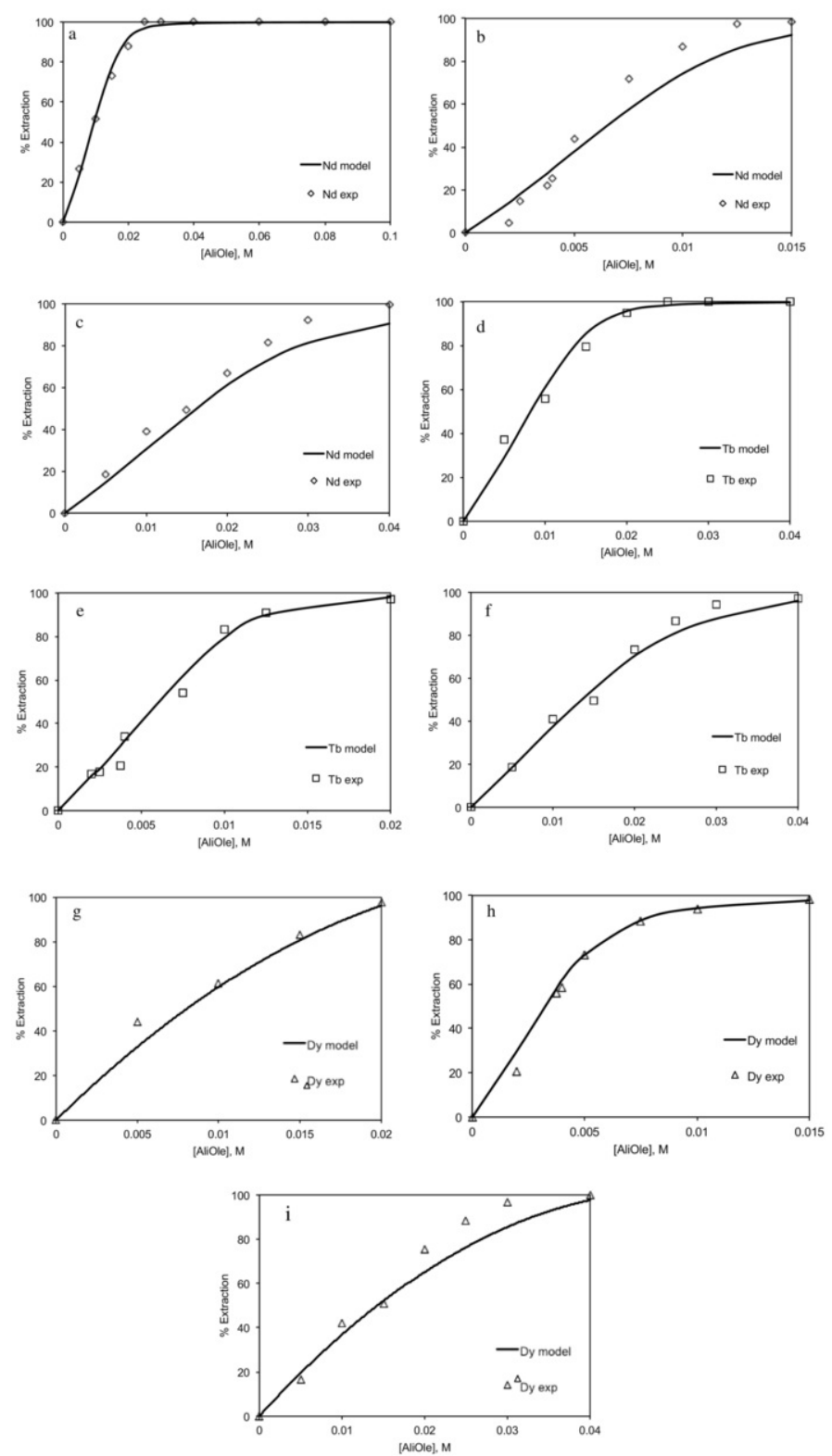

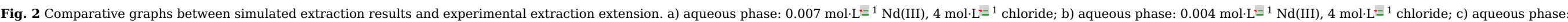

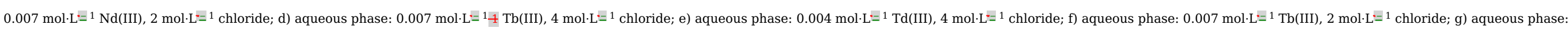
$0.006 \mathrm{~mol} \cdot \mathrm{L}=1$ Dy(III), $4 \mathrm{~mol} \cdot \mathrm{L}=1$ chloride; h) aqueous phase: $0.001 \mathrm{~mol} \cdot \mathrm{L}=1$ Dy(III), $4 \mathrm{~mol} \cdot \mathrm{L}=1$ chloride; i) aqueous phase: $0.006 \mathrm{~mol} \cdot \mathrm{L}=1$ Dy(II), $2 \mathrm{~mol} \cdot \mathrm{L}=1 \mathrm{chloride}$.

alt-text: Fig. 2 


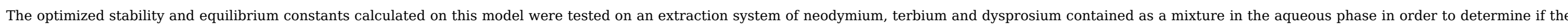

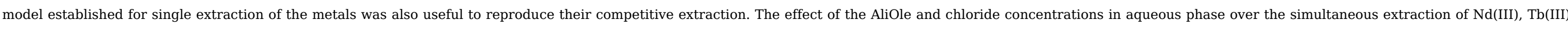
and Dy(III) was studied. Figure. 3 shows the comparative graphs between the simulation results of the model and the extraction percentages obtained experimentally.
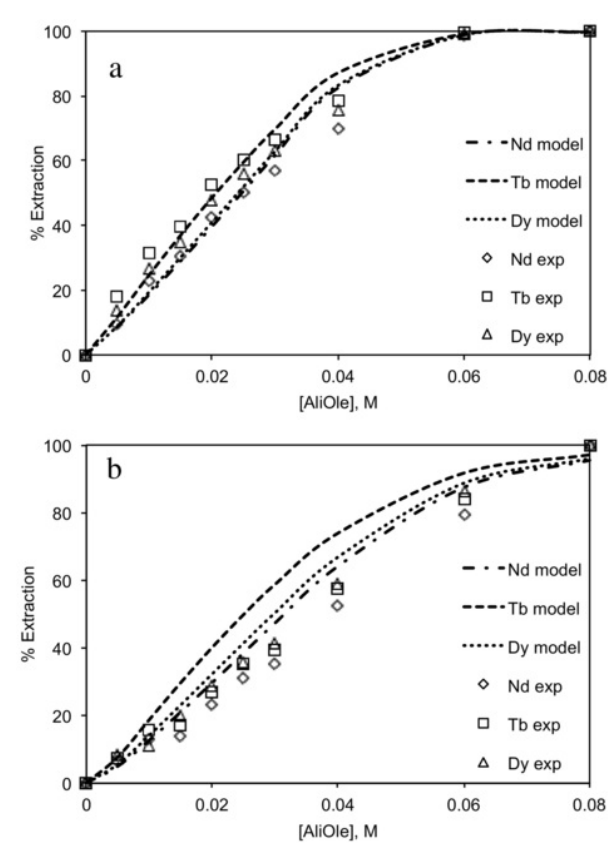

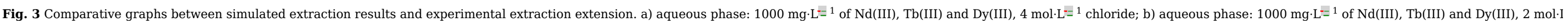
$\equiv 1$ chloride.

\section{alt-text: Fig. 3}

As shown, the equilibrium parameters calculated for the extraction of each metal separately allowed us to recreate feasibly the simultaneous extraction of Nd(III), Tb(III) and Dy(III) in the aqueous phase.

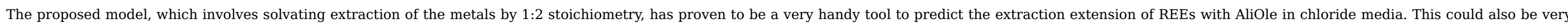

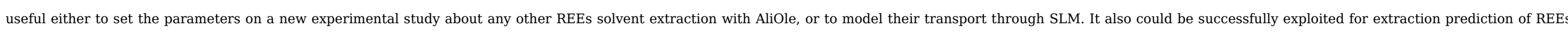
from end of life products (urban mining), requiring only minor adjustments of the equilibria equations and mass balances.

\section{6.}

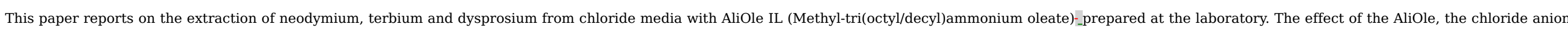

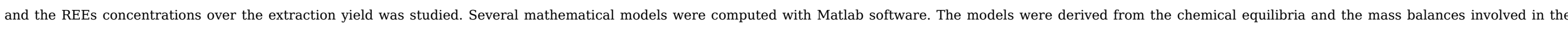

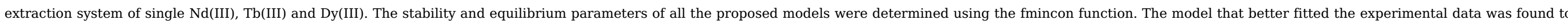

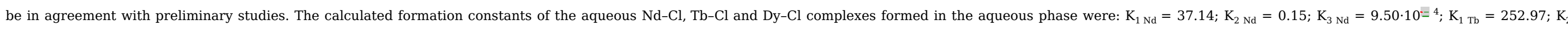

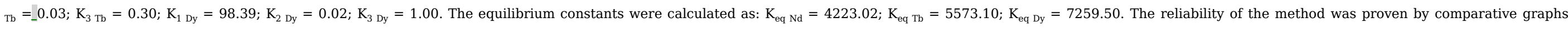
between the simulated model and the experimental extraction extension. 

by SLM.

\section{Acknowledgements}

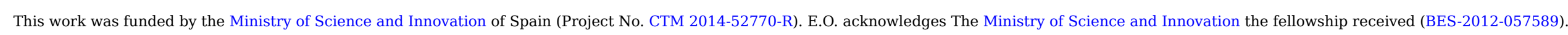

\section{References}

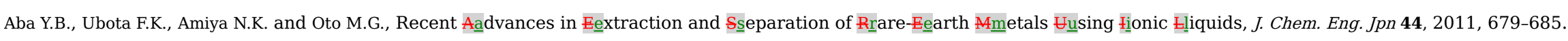

Baba Y., Kubota F., Kamiya N. and Goto M., Recent advances in extraction and separation of rare-earth metals using ionic liquids, J. Chem. Eng. 44, $2011,679-685$.

Coll M.T., Fortuny A. and Sastre A.M., Boron reduction by supported liquid membranes using ALiCY and ALiDEC ionic liquids as carriers, Chem. Eng. Res. Des. 92, 2014, 758-763,

https://doi.org/10.1016/j.cherd.2013.11.010.

Cossu R. and Williams I.D., Urban mining: Gconcepts, terminology, challenges, Waste Manag. 45, 2015, 1-3, https://doi.org/10.1016/j.wasman.2015.09.040.

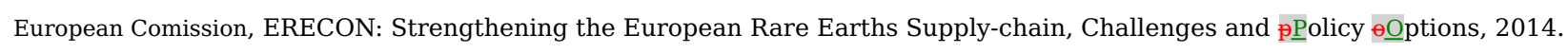

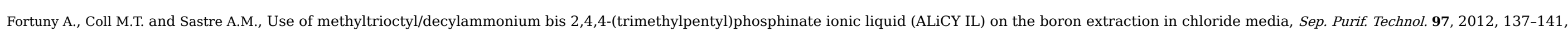
https://doi.org/10.1016/j.seppur.2012.02.037.

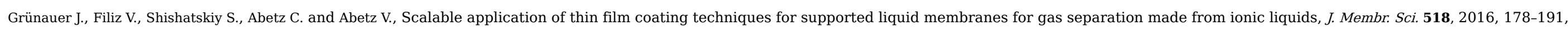
https://doi.org/10.1016/j.memsci.2016.07.005.

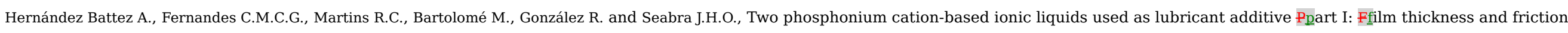
characteristics, Tribol. Int. 107, 2016, 233-239, https://doi.org/10.1016/j.triboint.2016.10.048.

Hogfeldt E., Stability Constants of Metal-1ion Complexes, Part A: Inorganic Ligands (IUPAC Chemical Data Series), 2nd ed., 1982, Pergamon Press.

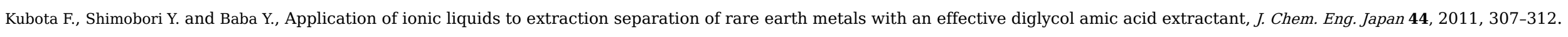

Lee M.-S., Lee J.-Y., Kim J.-S. and Lee G.-S., Solvent extraction of neodymium ions from hydrochloric acid solution using PC88A and saponified PC88A, Sep. Purif. Technol. 46, 2005, 72-78, https://doi.org/10.1016/j.seppur.2005.04.014.

Li Z. and Ren T., Synergistic effects between alkylphosphate-ammonium ionic liquid and alkylphenylborate as lubricant additives in rapeseed oil, Tribiol. Int. 109, 2017, 373-381, https://doi.org/10.1016/j.triboint.2016.11.032.

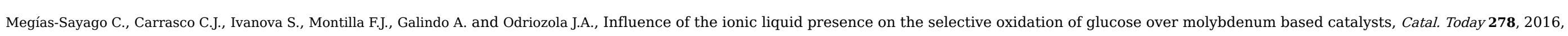
82-90, https://doi.org/10.1016/j.cattod.2016.06.040.

Mehrkesh A. and Karunanithi A.T., Optimal design of ionic liquids for thermal energy storage, Comput. Chem. Eng. 93, 2016, 402-412, https://doi.org/10.1016/j.compchemeng.2016.04.008.

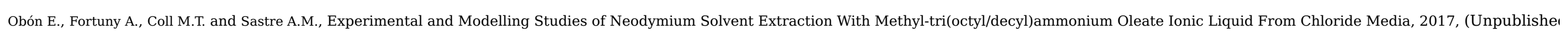
results).

Puigdomenech I., Medusa Software, 2013.

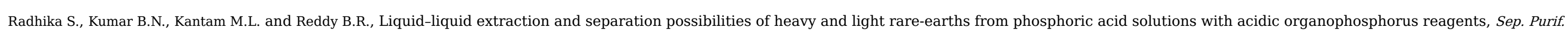
Technol. 75, 2010, 295-302, https://doi.org/10.1016/j.seppur.2010.08.018. 


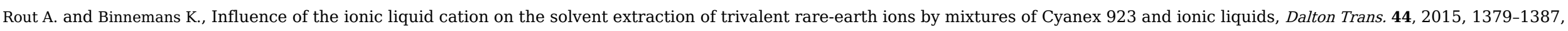
https://doi.org/10.1039/c4dt02766c.

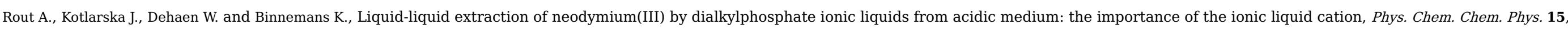
2013, 16533-16541, https://doi.org/10.1039/c3cp52218k.

Sastri V.S., Modern $¥ \underline{A}$ spects of $¥ \underline{R}$ are $e \underline{E}$ arths and their $€ \underline{C}$ omplexes, 2003, Elsevier.

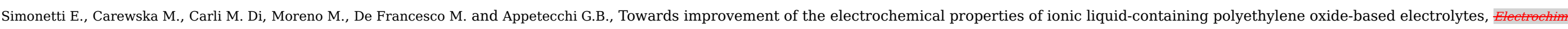
Acta.Electrochim. Acta 2017, https://doi.org/10.1016/j.electacta.2017.03.080.

Sun X., Bell J.R., Luo H. and Dai S., Extraction separation of rare-earth ions via competitive ligand complexations between aqueous and ionic-liquid phases, Dalton Trans. 40, 2011, 8019-8023, https://doi.org/10.1039/c1dt10873e.

Tunsu C., Ekberg C. and Retegan T., Characterization and leaching of real fluorescent lamp waste for the recovery of rare earth metals and mercury, Hydrometallurgy 144-145, 2014, 91-98, https://doi.org/10.1016/j.hydromet.2014.01.019.

U.S Department of Energy, Critical Materials Strategy, 2011.

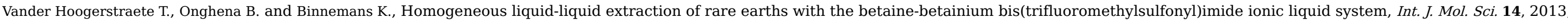
21353-21377, https://doi.org/10.3390/ijms141121353.

Vekariya R.L., A review of ionic liquids: Aapplications towards catalytic organic transformations, J. Mol. Liq. 227, 2017, 44-60, https://doi.org/10.1016/j.molliq.2016.11.123.

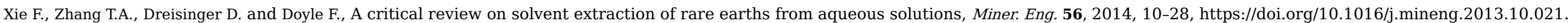

Zhang G.-R. and Etzold B.J.M., Ionic liquids in electrocatalysis, J. Energy Chem. 25, 2016, 199-207, https://doi.org/10.1016/j.jechem.2016.01.007.

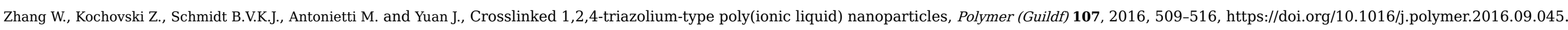

Zhu L., Guo L., Zhang Z., Chen J. and Zhang S., The preparation of supported ionic liquids (SILs) and their application in rare metals separation, SCIENCE CHINA Chem. 55, 2012, 1479-1487,

https://doi.org/10.1007/s11426-012-4632-8.

\section{Highlights}

- The mathematical model proposed is able to accurately reproduce the experimental data of $\mathrm{Nd}(\mathrm{III})$, $\mathrm{Tb}(\mathrm{III})$ and Dy(III) single extraction.

- Speciation of $\mathrm{Nd}(\mathrm{III}), \mathrm{Tb}(\mathrm{III})$ and $\mathrm{Dy}(\mathrm{III})$ in chloride media and competitive extraction of $\mathrm{HCl}$ are taken into account.

- The mathematical model proposed is able to predict the simultaneous extraction of $\mathrm{Nd}(\mathrm{III})$, $\mathrm{Tb}(\mathrm{III})$ and $\mathrm{Dy}(\mathrm{III})$.

\section{Queries and Answers}

\section{Query:}

"Table 1" has been changed to "Table 2". Please check if correct and amend as necessary.

Answer: It is correct 


\section{Query:}

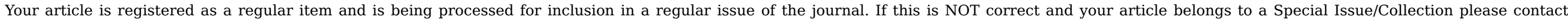
b.mahesh@elsevier.com immediately prior to returning your corrections.

Answer: Yes

Query:

Please confirm that given names and surnames have been identified correctly and are presented in the desired order, and please carefully verify the spelling of all authors' names.

\section{Answer: Yes}

Query:

The author names have been tagged as given names and surnames (surnames are highlighted in teal color). Please confirm if they have been identified correctly.

Answer: Yes

Query:

Highlights should only consist of 125 characters per bullet point, including spaces. The highlights provided are too long; please edit them to meet the requirement.

Answer: The highlights have been edited.

Query:

Please provide an update for reference "Obón et al., unpublished results".

Answer: Still not published 